ÉTUDES

DE STYLISTIOUE

ANGLAISE

\section{Études de stylistique anglaise}

$12 \mid 2018$

La Société de Stylistique Anglaise (1978-2018) : 40 ans de style

\title{
Le reste persiste et signe. Quelques petits problèmes de grammaire anglaise (ir)résolus
}

\section{Monique De Mattia-Viviès}

\section{(2) OpenEdition \\ Journals}

Édition électronique

URL : http://journals.openedition.org/esa/516

DOI : 10.4000/esa.516

ISSN : 2650-2623

Éditeur

Société de stylistique anglaise

Édition imprimée

Date de publication : 30 janvier 2018

Pagination : 143-176

ISSN : 2116-1747

Référence électronique

Monique De Mattia-Viviès, «Le reste persiste et signe. Quelques petits problèmes de grammaire anglaise (ir)résolus », Études de stylistique anglaise [En ligne], 12 | 2018, mis en ligne le 19 février 2019, consulté le 14 septembre 2019. URL : http://journals.openedition.org/esa/516; DOI : 10.4000/esa.516 


\title{
Le reste persiste et signe. Quelques petits problèmes de grammaire anglaise (ir)résolus
}

\author{
Monique DE MATTIA-ViviÈs \\ Aix Marseille univ, LERMA, Aix-en-Provence, France
}

À Jean-Jacques Lecercle, Gilles Mathis et Wilfrid Rotgé ${ }^{1}$

\begin{abstract}
Dans cet article d'hommage à la stylistique, j'oserai employer ce «je» de l'énonciation que la stylistique, en tant que discipline dont les contours ne sont pas figés, autorise.

Etre grammairien et stylisticien, c'est, selon moi, accepter l'irrésolu, accepter que certains problèmes de grammaire ne puissent s'expliquer dans le cadre d'une formalisation donnée, à un moment donné. Ceci n'est nullement une facilité. C'est un constat, imposé par l'instabilité constitutive de la langue. L'ombre de Jean-Jacques Lecercle et sa théorie du reste planent, on l'aura compris ${ }^{2}$. Mais si le grammairien stylisticien
\end{abstract}

1 Je remercie également mes amies stylisticiennes, Nathalie Vincent-Arnaud et Simone Rinzler, respectivement vice-présidente de la Société de Stylistique Anglaise (SSA) et présidente adjointe / co-rédactrice en chef de la revue Etudes de Stylistique Anglaise (ESA) lorsque je présidais cette société (2007-2011). Nos échanges fructueux et leur dévouement sans faille ont largement contribué à faire de mon passage à la tête de la Société un moment d'entente intellectuelle et amicale unique. Mes remerciements s'adressent également à Manuel Jobert, alors trésorier de la SSA, qui m'a ensuite succédé à la tête de la société (2011-2015), ainsi qu'à toute l'équipe qui m'a entourée. Je tiens enfin à adresser mes remerciements chaleureux à Sandrine Sorlin, actuelle présidente de la SSA, qui est à l'initiative de ce numéro-anniversaire.

2 Dans les termes de Jean-Jacques Lecercle, "la relation entre la grammaire et le reste n'est pas d'opposition ni d'inversion, mais d'excès. Décrire le fonctionnement du reste, ce n'est pas décrire des opérations différentes de celles de la grammaire, mais les mêmes opérations poussées à l'excès. Pour franchir la frontière, il faut prendre la route qui y mène, et simplement oublier de s'arrêter à la barrière. Parcours excessif, mais sur la même 
peut accepter l'irrésolu, il ne s'en accommode pas aisément pour autant. Bien au contraire, il cherche, inlassablement, LA solution, LA formalisation adéquate qui lui permettrait de rendre compte de tel ou tel phénomène, jusqu'à parfois se confronter à un obstacle, qui le dépasse : la langue, et son potentiel créatif. Si l'irrésolu est parfois le point d'arrivée inconfortable de son analyse, il n'en est jamais le point de départ. Postuler un échec possible de la formalisation n'est en aucun cas une commodité. Prendre pour objet le discours rapporté dans le roman moderniste par exemple (notamment dans certains romans de Virginia Woolf), c'est se confronter à nombre d'énoncés indécidables, mettant en échec le linguiste désirant établir une typologie parfaitement claire des formes de discours rapporté. La tâche conduit à la simplification ou à l'impasse si l'indécidable n'est pas accepté comme un travail de style.

Dans ces conditions, l'irrésolu, sorte de reste du travail de la langue et de sa prolifération, loin d'être un constat d'échec, a sa place dans une formalisation fondée sur une conception évolutive de la langue. Plus qu'un concept, le reste est une forme de pensée, qui a abouti, pour ce qui me concerne, à assigner une place à l'indécidable, à en faire un cas de figure spécifique au plan linguistique, comme résultant souvent, dans les cas que j'ai étudiés, d'une déconnexion forme / sens. Cette conception plastique du langage m'a conduite à mettre au jour une syntaxe qui ne dit pas toujours la vérité, une syntaxe qui se déconnecte du sens, une syntaxe mensongère. La stylistique est le lieu d'analyse privilégié de cette déconnexion forme /sens, le lieu où la théorie du signe de Saussure se défait, où le lien (ou point de capiton ${ }^{3}$ ) entre signifiant et signifié ne va plus de soi. En psychanalyse, cette déliaison est au cœur de l'écoute : ce que je dis n'est pas ce que je dis. En stylistique, la forme que prend le dire ne correspond pas toujours à ce qui est dit. Le sens construit par la forme parfois se défait, à un certain niveau, pour créer un sens nouveau. C'est à cette absence d'adhérence, partielle ou totale entre la forme et le sens, qui prend racine dans la notion stylistique d'écart, que j'ai consacré mes travaux linguistiques, et qui débouche sur un autre domaine d'investigation, la psychiatrie. La forme et le sens s'accordent ou se délient, ou les deux à la fois. Et la déliaison fait vaciller la grammaire, comme elle peut être le symptôme d'un vacillement de l'être. C'est en ce

route. [...] Le reste est le royaume des monstres de la langue — des fausses unités et des constructions illicites. " ([1990] 1996, 67).

3 J'emprunte cette formule à Jacques Lacan (1981). 
point de vacillement que la recherche peut prendre sens et progresser, sans crainte de s'enfermer dans les certitudes d'une théorie, quelle qu'elle soit.

J'évoquerai ici quelques petits problèmes de grammaire qui ont été abordés en fonction de cette conception de la stylistique comme lieu possible d'investigation du reste de la langue, prenant la forme de déconnexions forme/sens. Si l'analyse grammaticale échoue parfois, elle réussit pourtant si elle fait apparaître comme volontaire l'indécidabilité, signe d'une instabilité constitutive de la langue.

Plus précisément, les problèmes évoqués ci-dessous constituent chacun à sa manière des illustrations de ce reste. Ils sont à mes yeux soit indécidables au sens positif du terme, l'indécidable étant intentionnellement généré par un style d'écriture, soit mettent en péril une formalisation trop strictement appliquée, tordant le cou à la langue, dans sa plasticité et son évolution, soit aboutissent à des formes de déconnexion forme / sens qui produisent un autre sens que celui auquel la forme est attachée. Ils ont été choisis pour leur mise en évidence à la fois du travail du grammairien stylisticien, qui cherche, jusqu'à parfois aboutir à une solution multiple, à des cas indécidables jusqu'à l'irrésolu, parce qu'ils mettent en évidence la primauté de la sémantique sur la syntaxe, et induisent un rapport à la forme distancié, cette dernière n'en finissant pas de créer des sens inattendus.

Le positionnement étant donc celui du chercheur grammairien stylisticien qui souvent tâtonne, parfois trouve, mais toujours accepte l'incomplétude, voire l'erreur, due au travail de la langue elle-même, ce qui l'amène à revoir ses propres analyses. Chercher, c'est écrire et réécrire, sans que cette réécriture ne soit nécessairement le signe d'une erreur préalable. La langue travaille, y compris à l'intérieur de soi et à son insu. D'où l'évolution d'une position, qui ne peut sans crainte être reconnue qu'à l'intérieur d'un cadre qui accepte la stable instabilité de la langue, et en fait son point de départ.

\section{Noir, c'est noir, and must is (not) must. De l'excès de sens}

On se souvient de la célèbre première phrase de Pride and Prejudice de Jane Austen, à laquelle Jean-Jacques Lecercle consacre une analyse dans son ouvrage The Violence of Language (1990, 12-14) :

It is a truth universally acknowledged, that a single man in possession of a good fortune must be in want of a wife. 
Cet énoncé fait apparaître une contradiction, qui semble provenir de l'ambiguïté de l'auxiliaire de modalité must dans une telle configuration syntaxique. Must peut ici en effet recevoir deux interprétations, radicale ou épistémique, fait relativement rare avec ce modal au sein d'un même énoncé; généralement, le contexte permet de trancher entre la valeur radicale ou la valeur épistémique :

- il peut signifier que tout homme célibataire in possession of a good fortune se doit d'être à la recherche d'une femme. Une obligation morale, imposée par la société, et non par la narratrice, pèse sur lui. Dans ce cas, must a une valeur radicale déontique.

- Il peut également signifier qu'il est logique qu'un homme célibataire in possession of a good fortune soit à la recherche d'une femme. Dans ce cas, must a une valeur épistémique et renvoie à une probabilité logique. On se situe alors très haut sur le gradient confidence /doubt de Coates (1983).

Dans les deux cas, on a affaire à de la modalisation a priori (Larreya 2000 ), portant sur la validation de la relation prédicative (RP), soit en termes de chances de validation, soit en termes d'obligation portant sur le sujet de l'énoncé. De façon schématique, la modalisation $\boldsymbol{a}$ priori renvoie à un procès non validé, que la modalité soit radicale déontique (you must stop immediately) ou épistémique (he must have lied). La modalisation a posteriori quant à elle suppose une RP validée (It can be very cold in Scotland). Elle intervient donc après coup, à partir d'une RP déjà validée. Le concept de modalisation a priori permet un rapprochement entre modalités épistémique et modalité radicale déontique sur lequel on reviendra plus loin puisque dans les deux cas, les procès concernés sont virtuels, la validation de la RP n'ayant pas eu lieu.

Revenons sur l'interprétation épistémique de must : celle-ci semble entrer en contradiction avec le début de l'énoncé (proposition matrice) : It is a truth universally acknowledged. En effet, si le fait qu'un riche célibataire soit à la recherche d'une femme est une vérité admise de tous, l'on s'attend à ce que la relation prédicative soit présentée comme validée : It is a truth universally acknowledged, that a single man in possession of a good fortune is (always) in want of a wife. L'on se demande alors pourquoi cette dernière serait présentée comme relevant d'une probabilité logique, donc comme faisant l'objet d'un doute. Cette interprétation estelle alors réellement légitime? Doit-elle être écartée au profit de 
l'interprétation radicale déontique ? Il semble qu'elle résiste ou qu'elle insiste pourtant et qu'elle puisse se soutenir si l'on part du principe que that a single man in possession of a good fortune must be in want of a wife correspond implicitement à du discours rapporté, et que la matrice, it is a truth universally acknowledged, dissimule sous son caractère générique, une source énonciative différente de celle de la subordonnée. Dans une telle interprétation, l'on n'aurait nullement affaire à une assertion (contradictoire) de la narratrice qui d'abord asserte l'existence d'un fait admis de tous pour ensuite présenter cet objet comme faisant l'objet d'un doute, mais à l'expression d'un point double point de vue, celui de la narratrice, logé dans la matrice, et celui d'un personnage, dont on comprend très vite qu'il s'agit de celui de Mrs Bennet, logé dans la subordonnée.

En d'autres termes, si la syntaxe semble légèrement contradictoire dans une interprétation épistémique du modal, c'est parce que la généricité de la matrice s'oppose à la spécificité du doute : une incertitude portant que la validation d'une RP ne peut être universellement admise. Au niveau lexical, acknowledge, verbe factif ${ }^{4}$, présupposant que le contenu qu'il introduit est vrai pour le locuteur, est contradictoire avec l'expression d'un doute dans la subordonnée. Le hiatus est là, dans ce conflit apparent, qui ne se présente pas explicitement comme tel. On ne comprend qu'ensuite que la matrice is a truth universally acknowledged est ironiquement assertée par la narratrice et constitue un commentaire ironique de cette dernière sur le contenu de la subordonnée, et que cette vérité universellement admise ne l'est qu'au sein d'un village, et à l'intérieur de celui-ci, par Mrs Bennet elle-même (universally se réduisant ainsi successivement à the minds of the surrounding families, puis implicitement à Mrs Bennet). Quant à la subordonnée extraposée, il apparaît grâce au paragraphe suivant qu'elle reflète le point de vue de Mrs Bennet (comme le montrent les éléments en gras, qui contiennent des modalités épistémiques et radicales évoquant l'ambiguïté du MUST du premier énoncé) :

"Is he married or single?"

"Oh! Single, my dear, to be sure! A single man of large fortune; four or five thousand a year. What a fine thing for our girls!"

"How so? How can it affect them?"

\footnotetext{
$4 \quad$ Terme emprunté à Kiparsky (1971).
} 
"My dear Mr. Bennet," replied his wife, "how can you be so tiresome! You must know that I am thinking of his marrying one of them." "Is that his design in settling here?"

"Design! Nonsense, how can you talk so! But it is very likely that he MAY fall in love with one of them, and therefore you must visit him as soon as he comes."

Cette contradiction ne peut donc s'expliquer que par le fait que les deux propositions ne sont pas soutenues par la même source énonciative.

Cet effet de hiatus ne se produit pas dans l'interprétation radicale car énoncer une obligation provenant de la société portant sur le sujet de l'énoncé (bien qu'a priori l'expression be in want of a wife se prête mal à une obligation), n'est pas contradictoire avec le fait qu'elle soit admise de tous. Même si dans l'interprétation radicale déontique de must, la subordonnée relève aussi du point de vue de Mrs Bennet (un homme riche se doit d'être à la recherche d'une femme, se doit d'avoir besoin d'une femme, c'est en tout cas l'obligation que Mrs Bennet veut croire que la société lui impose), il n'y a pas d'effet de hiatus car la modalité contenue dans la subordonnée (déontique, axe bon/mauvais) ne contredit pas celle de la principale (axe vrai/faux). Les deux axes sur lesquels elles opèrent sont différents. En revanche, dans l'interprétation épistémique, acknowledge renvoie au certain (axe vrai/faux) alors que must renvoie à de l'incertain (axe vrai/faux également). C'est le même axe qui est concerné, la matrice venant contredire la subordonnée.

Une obligation est plus facilement universellement admise qu'une probabilité logique.

Ce must est donc indécidable et renvoie à un excès de sens, à un reste dans les termes de Lecercle (1990, 12-14), qui n'est pas prévu par la grammaire de ce modal, qui a normalement un sens radical ou un sens épistémique, le contexte permettant généralement de lever toute ambiguïté.

Cet excès de sens a pour conséquence que modalités radicale et épistémique ici se rejoignent. En d'autres termes, je proposerais l'hypothèse suivante : dans une telle configuration syntaxique (must + la copule be dans l'expression de sens statique be in want of), le modal a les deux sens : il renvoie à la fois à une obligation morale qui, selon Mrs Bennet, doit peser sur any single man in possession of a good fortune (car 
tel est son désir à elle, qui a cinq filles à marier), et à une probabilité/nécessité logique, étant donné qu'un homme riche doit toujours selon Mrs Bennet et selon toute logique, chercher une femme à épouser. Cette dualité de sens provient du fait que must véhicule une modalisation a priori, qu'elle soit épistémique ou radicale. Dans les deux cas, la modalité porte sur la RP et sa validation non effective. Les valeurs véhiculées par le modal se rejoignent et se confondent. Le signifiant must incorpore alors ses deux signifiés, d'où l'excès.

Cette analyse diffère donc de celle de Lecercle, pour qui l'excès de sens provenait ici, à grands traits, d'un emploi radical transgressif du modal must dans ce contexte (la locution be want of a wife ne saurait en effet faire l'objet d'un ordre, et pourtant c'est bien ainsi qu'il faut interpréter le modal), faisant double-emploi avec la formule initiale it is a truth universally acknowledge $\bar{\varphi}$. Mais si mon analyse diffère, elle conforte pourtant sa théorie du reste. Elle constitue l'illustration du travail du reste, qui continue inlassablement de faire son œuvre. La nécessité logique et la nécessité déontique se rejoignent en tant que modalisations a priori, jusqu'à, dans certains contextes, devenir indistinctes.

Cette ambiguïté débouchant sur un double sens se retrouve d'ailleurs en français :

Il est universellement admis qu'un homme célibataire possédant une fortune assez confortable doit/doive être à la recherche d'une femme.

Et l'on constate en outre que must, non précédé de la matrice, conserve son double sens:

A single man in possession of a good fortune must be in want of a wife.

L'énoncé serait alors interprété comme du discours indirect libre (DIL), reflétant le point de vue de Mrs Bennet, mais il n'y aurait plus de hiatus syntaxique. La source serait homogène.

Cet énoncé fait donc apparaître un double problème: celui de l'interprétation de must, et l'effet de hiatus qu'il produit.

Mais ce hiatus ne provient pas du double sens de must, que ce dernier conserve si l'on omet la matrice. Il provient précisément du

5 La pensée de Lecercle est plus complexe et ne saurait se résumer à cette présentation très synthétique. On renverra donc à son analyse, sur laquelle la mienne prend largement appui (1996 [1990], 15-17). 
double point de vue que l'énoncé recèle : celui de la narratrice, ironique, logé dans la matrice, et celui de Mrs Bennet, logé dans la subordonnée.

Le hiatus est ainsi un effet de syntaxe, crée par une source énonciative non homogène, qui vient s'ajouter à l'excès de sens du modal dans ce cas.

C'est donc la configuration syntaxique polyphonique qui génère un conflit, non le modal lui-même, qui par son double sens, contribue à l'excès et offre un cas de figure non prévu par la grammaire. Mais les types de modalité ne s'opposent pas et se rejoignent parfois. En outre, elles s'incarnent ici dans le même signifiant.

Cet emploi de must offre ainsi une figure de l'indécidable. Il a les deux sens à la fois.

\section{Lost in syntax}

L'analyse syntaxique n'épuise pas la description du fait syntaxique.

(Philippe 2016, 236)

Comment décrire l'énoncé en gras ci-dessous ? Ce dernier est en apparence complexe au plan syntaxique, mais produit un effet de brouillage : comment distinguer les propositions qui le composent?

The Ministry of Truth contained, it was said, three thousand rooms above ground level, and corresponding ramifications below. Scattered about London there were just three other buildings of similar appearance and size.

So completely did they dwarf the surrounding architecture that from the roof of Victory Mansions you could see all four of them simultaneously. They were the homes of the four Ministries between which the entire apparatus of government was divided. The Ministry of Truth, which concerned itself with news, entertainment, education, and the fine arts. The Ministry of Peace, which concerned itself with war. The Ministry of Love, which maintained law and order. And the Ministry of Plenty, which was responsible for economic affairs. Their names, in Newspeak: Minitrue, Minipax, Miniluv, and Miniplenty. (Orwell, 1984,6)

On remarque que le groupe adverbial so completely a été antéposé, so déclenchant l'inversion sujet / auxiliaire, so completely did they dwarf... C'est comme si l'énoncé n'était constitué que de propositions 
subordonnées, comme si so subordonnait le premier segment, et that le deuxième :

So completely did they dwarf the surrounding architecture. (subordonnée 1)

that from the roof of Victory Mansions you could see all four of them simultaneously. (subordonnée 2)

Il est ici utile de restaurer l'ordre canonique SVO :

They $d$ warfed the surrounding architecture so completely that from the roof of Victory Mansions you could see all four of them simultaneously.

Il apparaît que l'énoncé comporte une proposition subordonnée affectant l'adverbe completely: so [completely] that from the roof of Victory Mansions you could see all four of them simultaneously, et que cette dernière est composée de l'adverbe de degré so corrélé à la proposition de conséquence introduite par la conjonction that. So... that fait donc office de conjonction corrélée, et l'ensemble constitue une proposition adverbiale de degré/conséquence portant sur l'adverbe completely. A l'intérieur de la subordonnée corrélative portant sur completely, on trouve un groupe prépositionnel (GPrep) complément circonstanciel de lieu, from the roof of Victory Mansions, portant sur la $\mathrm{RP}$ qui suit, you could see all four of them simultaneously.

Si l'on considère que pour être syntaxiquement complexe, un énoncé doit contenir une proposition subordonnée portant sur la RP de la principale, l'on n'a pas ici affaire à un énoncé complexe, la portée de la corrélative étant réduite à l'adverbe completely.

On avancera donc qu'au plan strictement syntaxique, on a affaire à un énoncé simple, réduit à une seule proposition They dwarfed the surrounding architecture completely qui incorpore une proposition adverbiale corrélée, so ... that from the roof of Victory Mansions you could see all four of them simultaneously dont la portée est limitée à un seul élément, l'adverbe completely. Cette adverbiale est complément circonstanciel de l'adverbe completely et pourrait être remplacée par un adverbe, par exemple almost: They dwarfed the surrounding architecture almost completely. Elle ne complexifie donc pas l'énoncé lui-même.

De ce fait, l'énoncé est au plan syntaxique, faussement complexe : il contient un groupe adverbial complexe complément circonstanciel de 
manière, mais l'adverbiale n'est pas subordonnée à une principale même si elle produit cet effet.

La structure fonctionnelle de l'énoncé de l'énoncé est donc S (They) V (dwarfed) O (the surrounding architecture) CCManière (so completely that from the roof of Victory Mansions you could see all four of them simultaneously).

Pourtant, cette analyse purement syntaxique est contre-intuitive : l'analyse syntaxique n'épuise pas la description du fait syntaxique, pour reprendre la formule de Philippe $(2016,236)$.

L'analyse sémantique fait apparaître un hiatus entre la syntaxe et le sens.

Le sens introduit une véritable hiérarchie sémantique entre deux propositions dont l'une dépend de l'autre, alors que l'énoncé est syntaxiquement simple :

Because they dwarfed the surrounding architecture completely

from the roof of Victory Mansions, you could see all four of them simultaneously

Il apparaît qu'au plan du sens, ce qui est principal et a le plus de poids, you could see all four of them simultaneously, correspond à la proposition subordonnée au plan syntaxique. Inversement, ce qui est principal au plan syntaxique apparaît comme secondaire au plan sémantique : they dwarfed the surrounding architecture completely.

Il ressort donc que la proposition qui est syntaxiquement subordonnée à l'adverbe est ici sémantiquement principale, et que la proposition syntaxiquement principale, qui incorpore la subordonnée corrélative portant sur l'adverbe, est sémantiquement subordonnée, c'està-dire non porteuse de l'information principale. La proposition syntaxiquement adverbiale, en théorie sémantiquement secondaire, véhicule l'information principale. L'ordre thème/rhème, qui fait apparaître deux informations, donc deux propositions (So completely did they dwarf the surrounding architecture // that from the roof of Victory Mansions you could see all four of them simultaneously) ne correspond pas à la hiérarchie syntaxique (une seule proposition, qui en englobe une autre, portant non sur une RP mais sur un constituant unique de cette proposition englobante). 
La structure syntaxique de cet énoncé (simple) est donc contredite par sa structure sémantique (complexe, faisant apparaître une hiérarchie), la proposition syntaxiquement principale étant sémantiquement subordonnée. L'ordre des mots ainsi que le découpage syntaxique proposition principale / proposition subordonnée ne correspond pas au poids sémantique des propositions. La structure syntaxique de l'énoncé est contredite par sa structure sémanticothématique. Le hiatus mis au jour fait apparaître la prééminence du sens sur la syntaxe, qui a toujours le dernier mot.

Une analyse plus stylistique, fondée sur le sens et les phénomènes de déconnexion, permet donc de mettre en lumière ce type de contradiction apparente : ce qui est principal au plan syntaxique ne l'est pas toujours au plan sémantique.

\section{Paradoxe}

Rivara dans Le système de la comparaison (1990, 168-183), s'intéresse aux énoncés du type John claims he is cleverer than he is, Boris said he didn't kiss the girl he kissed.

Prenons le premier exemple : cet énoncé relève de ce que l'on appelle communément le discours indirect classique (DIC). Ce dernier se caractérise le plus souvent par la présence d'une proposition matrice du type $X$ says/said, ici John claims, et d'une proposition nominalisée complétive, ici sans that, he is taller than he is, objet de claim. On note que cette dernière incorpore une proposition comparative corrélée, -er than he is. Au plan temporel, on constate une déconnexion entre time et tense : le contenu de claim renvoie à un acte d'énonciation passé (time, temps notionnel), mais ce dernier est toujours présent aux yeux du locuteur, d'où l'emploi du présent (tense, temps grammatical).

Une première constatation : la conjonction de subordination that est absente, et ne pourrait aisément être employée (les différents locuteurs natifs interrogés sont en effet très partagés), ? John claims that he is taller than he is. Deuxième constatation : l'énoncé produit un effet paradoxal : "tout se passe comme si [...] l'énonciateur attribuait au locuteur cité une énonciation contradictoire" (Rivara 1990, 168) du type *I'm taller than 1 am tall. L'origine énonciative de la comparative, -er than he is, semble 
donc poser problème et être différente de celle qui sous-tend he is tall. Or l'objet du verbe matriciel reflète généralement, en DIC, un point de vue unique, celui du locuteur cité.

Si l'on tente de reconstruire un discours origine ou génodiscours ${ }^{6}$ à partir de l'énoncé analysé, ce dernier pourrait par exemple correspondre à :

I'm tall.

I'm tall enough to play basketball.

I'm really / exceptionally tall.

I'm taller than you (are).

Mais en aucun cas, cet énoncé ne pourrait correspondre à ${ }^{\star} I$ 'm taller than I am.

L'énoncé John claims he is taller than he is semble donc réaliser l'articulation de deux points de vue : celui du locuteur cité (par exemple "I'm tall enough to play basketball ") et celui du citant, qui y adjoint un commentaire (par exemple he's not so tall, prenant la forme d'une comparative, -er than he is), produisant cet effet apparemment contradictoire. En d'autres termes, la proposition comparative corrélée, -er than he is est nécessairement le fait du locuteur citant. D'où l'étiquette structure corrélative apparemment anomale attribuée par Rivara à ce type de comparative corrélée à l'intérieur d'une complétive. Cette analyse rejoint celle des sémanticiens générativistes (par exemple Mc Cawley 1973, 217-231). Ces derniers soulignent que le contenu de la subordonnée corrélative doit échapper à la portée du verbe déclaratif pour que ces énoncés aient un sens, et proposent la notion "d'origine externe de la subordonnée ".

Un lien semble pouvoir être établi entre l'absence de that et l'effet polyphonique produit par la subordonnée. C'est la comparative corrélée à l'intérieur de la complétive articulant deux points de vue différents qui semble faire obstacle à un emploi non problématique :

John claims he is taller than he is.

? John claims that he is taller than he is.

\footnotetext{
6 J'emprunte ce terme à Philippe (1997).
} 
That redevient possible en l'absence d'une corrélée :

John claims that he is tall

Titor unambiguously claimed that the last Olympics would occur in 2004. While it is unclear if he meant the summer or winter Olympics, both have occurred since this time.

L'énoncé ?John claims that he is taller than he is semble attribuer au locuteur cité une pensée contradictoire : en tant que "marqueur de prise en charge différée $\rrbracket^{7}$ servant à repérer ce qui le suit dans la sphère du sujet de l'énoncé, ici John, locuteur cité, la présence de that, qui renvoie à l'acte initial, suppose généralement que l'intégralité de la subordonnée puisse être attribuée au locuteur cité. D'où la contradiction produite par son emploi, et le fait que claim $\varnothing$ soit logiquement préféré dans ce cas. L'effet polyphonique ne peut aisément être produit qu'en l'absence de that, claim $\varnothing$ ayant ici une valeur contrefactive ${ }^{8}$ : pour le locuteur citant, John se trompe sur sa taille.

On accepterait d'ailleurs, dans cette même valeur contrefactive,

John claims to be taller than he is.

$T o$, de par la dévirtualisation dont il est la trace, peut référer à des procès dont la validation n'a pas abouti ou fait l'objet d'un doute. En conséquence, claim $\varnothing$ semble donc plus proche de claim toV que de claim that.

Cet énoncé apparemment contradictoire ne peut se comprendre si l'on part du principe qu'il ne peut y avoir de déconnexion dans l'unité du sujet parlant (voir Banfield et sa théorie du 'one speaker / one self'). Un énoncé fait entendre un point de vue, celui du locuteur. Une conception dialogique ou polyphonique du discours rapporté et du langage en général (voir par exemple les analyses de Bakhtine (1926) et de Ducrot (1984)) est seule à même de rendre compte de l'imbrication de points de vue, et fait également apparaître la prééminence de la sémantique sur la syntaxe. Des énoncés comme Boris said he didn't kiss the girl he kissed ne

Terme employé par Chuquet et Bouscaren (1987, 175).

Terme employé par Kiparsky (1971). 
peuvent être interprétés correctement que si une partie de la complétive représente la pensée du locuteur citant, et non uniquement celle du cité. Ce qui est subordonné n'est donc pas attribuable à une seule source.

Toutes les théories de la polyphonie se fondent sur l'idée qu'il y a une rupture ou déconnexion dans l'unité du sujet parlant. Cette rupture m'amènera plus loin à la rupture dans l'unité du sujet pensant.

\section{Prise de « tête "}

Certains linguistes (par exemple Cotte, 1983) se sont intéressés à la structure suivante :

an angel of a girl, a fine figure of a man/woman, a gem of a place, a monster of a wife, his drunkard of a father, your idiot of a brother, a haunting beauty of a play, her pocket-handkerchief of a lawn, conceptualisée sous la forme N1 of N2.

Cette structure fait apparaître une hiérarchie, même si en apparence deux $\mathrm{N}$ sont reliés au moyen de of, qui semblent avoir un poids égal. On s'est notamment posé les questions suivantes : au plan syntaxique, of $\mathrm{N} 2$ complète-t-il N1, à la manière d'un complément du nom classique, $\mathrm{N} 1$, constituant au sein de la structure, le nom tête ? Au plan sémantique, of N2 constitue-t-il l'apport d'information ? Quel est le rôle de of: quelle est la nature du lien qu'il établit ? Indique-t-il une identité entre N1 et N2 ou marque-t-il une différence ? En tant que préposition, fait-il corps avec le GN qu'il introduit?

On examinera les énoncés suivants :

He's a gem of a person. (= someone who is very good and kind and goes out of his way to help other people)

The biggest man in the patrol, a giant of a man, lifted Jenny on to his shoulders.

She's a fine figure of a woman - not like all these skinny models. ( = someone who is big and strong with an attractive body)

Prenons He's a gem of a person. Cette configuration semble constituer une réorganisation synthétique de that person is a gem, aboutissant à la création d'un type de person, a gem of a person. La relation instaurée par of entre les deux $\mathrm{N}$ est de type identification : si he's a gem of a person constitue la réorganisation de that person is a gem, 
that person est qualifiée de gem par le biais de la copule be marquant une identification (ici métaphorique) entre les termes reliés.

$\mathrm{Au}$ niveau du sens, a gem qualifie a priori ou pré-qualifie le nom girl, comme le montre la glose that person is a gem. Le sujet, person, déjà connu, se trouve relégué en position de complément du nom dans une structure où of marque une identification entre gem, la qualification, et person, l'élément qualifié.

On a donc inversé le schéma qualifié (sujet, thème) / qualification (attribut du sujet, rhème) pour obtenir le schéma qualification/qualifié, rhème/thème :

That person is a gem : qualifié (sujet, thème)/ qualification (attribut du sujet, rhème)

$A$ gem of a person : qualification (N1) / qualifié (N2), rhème/thème.

Ce qui constitue l'apport sémantique ici est donc N1, gem, plus que $\mathrm{N} 2$, qui est présupposé par la présence de he.

En conséquence, le GPrep of a person n'est complément du nom angel qu'en surface car il n'apporte pas d'information nouvelle permettant de repérer gem (comme dans the trees of the avenue) : il constitue un rappel informatif, et opère un élargissement de la référence initiale (he), permettant d'aboutir à un type : persons who are gems.

A partir de là, le découpage suivant peut être effectué : He's a gem [of a person]. A gem est à la fois la tête syntaxique, c'est-à-dire le point d'appui sur lequel vient s'agréger of N2 (du moins en surface) et noyau sémantique du groupe, constituant l'apport d'information'. Of a person joue ici un rôle secondaire, permettant de faire de he un type.

On peut alors proposer la schématisation suivante :
A gem
of a person
Tête syntaxique
construction d'un type
Noyau sémantique

\footnotetext{
9 On pourrait avancer qu'en profondeur, c'est toujours N2 qui, dans ce type de structures, est la tête syntaxique du groupe : an angel of a girl = that girl is an angel.

Tête syntaxique / Apport sémantique

Toujours est-il qu'en surface, c'est N1 qui sert de point d'appui à N2.
} 
Of a person est suppressible, avec un léger changement de sens : he's a gem. Dans ce cas, l'on a une qualification unique, sans création d'un type générique.

Les deux autres énoncés cités se laissent analyser de la même manière.

Mais dans d'autres configurations de ce type, cette analyse ne semble pas correctement fonctionner :

Everything there in the warm kitchen [...] was good, dull, and sufficient. $[\ldots]$

"Everything's nice in that old hole," said Leslie, grumbling. It began to rain much more heavily. It bucketed down on the black backyard, and the little comfy kennel of a house [...]. (Thomas, "The Followers", 1980 [1955], 70)

Si la formalisation précédente est appliquée, comme on serait tenté de le faire s'agissant de la même structure, elle contrevient au sens du passage. Certes, une formalisation unique serait préférable à une formalisation qui s'adapte à chaque cas. Or la structure $\mathrm{N}$ of $\mathrm{N}$ empêche que lui soit appliquée une formalisation unique. Ou alors, cette dernière ne m'est tout simplement pas accessible à ce stade.

Dans cet extrait, il apparaît clairement que le nom house (N2) joue un rôle plus important.

Si kennel (N1) est en surface la tête syntaxique, servant de point d'appui au GPrep of a house, le noyau sémantique de l'ensemble semble dans ce cas correspondre à house, même si la qualification (little comfy kennel) constitue un apport d'information. La glose suivante semble conforter cette hypothèse :

It bucketed down in the black back yard and the house, which was like a little comfy kennel.

Of a house, contrairement à of a person, n'est pas ici suppressible.

En conséquence, suivant le sens des éléments en présence, et le caractère plus ou moins présupposé (donc syntaxiquement obligatoire) de N2, l'analyse peut être différente.

On avancera l'hypothèse que dans ce $2^{\text {ème }}$ cas de figure, tête syntaxique et noyau sémantique ne coïncident pas : la tête syntaxique, kennel, est en décalage partiel avec le noyau sémantique, house, qui joue 
un rôle plus important (même si the house n'est pas une information nouvelle). Le découpage sémantique serait alors le suivant: It bucketed down in the black back yard and the [little comfy kennel of a] house.

Prenons un autre exemple :

"Cissie" was the name of one of these villas, "Albert" of the other. These titles were not only picked out in shaded Gothic on the garden gates, but appeared a second time on the porches, where they followed the semicircular curve of the entrance arch in block capitals. "Albert" was inhabited. His tortured garden was bright with geraniums and lobelias and polished shells. His little windows were chastely swathed in Nottingham lace. "Cissie" was to let. Three notice-boards, belonging to Dorking agents, lolled on her fence and announced the not surprising fact. Her paths were already weedy; her pocket-handkerchief of a lawn was yellow with dandelions. (Forster, A Room with a View, 1908, 100)

Cet ensemble synthétique permet de poser à la fois le nom qualifié (lawn) et sa qualification (her pocket-handkerchief). Lawn entre dans une relation de comparaison avec pocket-handkerchief. Lawn (N2) est ici encore le noyau sémantique de la structure et pocket-handkerchief, la tête syntaxique (point d'appui à of N2). Ici encore, le même décalage entre tête syntaxique et noyau sémantique est constaté, même s'il reste partiel : pocket-handkerchief certes joue un rôle informatif mais il est second par rapport à lawn, qui ne peut être supprimé, alors que pocket-handkerchiet le peut : her [pocket-handkerchief of a] lawn was yellow with dandelions.

Même si l'existence de lawn est préparée par le contexte (il est mentionné que chaque maison a un jardin), et que l'on s'attend à son existence, il n'en reste pas moins que lawn constitue dans ce cas le noyau sémantique.

Le rôle prépondérant de $\mathrm{N} 2$ apparaît plus nettement lorsque cette structure est employée dans des contextes de première mention :

"Who said that?"

"Your fool of a husband."

Deux informations sont ici données, par ordre d'importance :

- c'est 'your husband' qui a dit cela

- 'your husband' est un imbécile.

$\mathrm{N} 2$ est donc le noyau sémantique. 
On note toutefois que dans la plupart des cas, la structure $N$ of $N$ ne s'emploie pas dans les cas de première mention; le lien effectué entre les deux $\mathrm{N}$ par le biais de of, suppose que N2 préexiste à la qualification :

"What is Big Sur, Mom?"

"?It's a gem of a place."

"It's a place in California. A gem of a place."

Dernier exemple : sur la couverture d'une édition numérique du livre Edward the Conqueror, de Roald Dahl (e-pub, 2012), on lit :

Edward the Conqueror is a brilliant gem of a short story from Roald Dahl, the master of the sting in the tail. In Edward the Conqueror, Roald Dahl, one of the world's favourite authors, tells a sinister story about the darker side of human nature. Here, a cat's curious behaviour comes between a husband and his wife . . . Edward the Conqueror is taken from the short story collection Kiss Kiss, which includes ten other devious and shocking stories $[\ldots]$.

Le contexte est définitoire, short story est le noyau sémantique (Edward the Conqueror is a short story), en décalage partiel avec la tête syntaxique, a brilliant gem, qui la qualifie (that short story is a gem). On dit à la fois ce qu'est Edward the Conqueror, à savoir a short story et on la qualifie de brilliant gem en même temps. La tête syntaxique qualifiante constitue un apport sémantique, mais sans doute moindre que $\mathrm{N} 2$, short story, même si ce terme constitue un attendu (il figure sur la couverture d'un livre), autorisant l'emploi en première mention de la structure dans ce contexte de définition.

On remarquera que dans les trois exemples illustrant le $2^{\text {ème }}$ cas de figure, le lien entre $\mathrm{N} 1$ et $\mathrm{N} 2$ ne va pas de soi : pocket-handkerchief ne s'associe pas aisément à lawn, de même que kennel n'est pas associé à house, ni gem à short story. D'où le fait que N2 joue ici un rôle plus important alors que dans le premier cas de figure envisagé, he's a gem of a person, gem peut plus aisément être associé à une personne. Of a person joue alors un rôle secondaire.

Deux hiérarchies sont donc possibles suivant le contexte et le lien cognitif entre $\mathrm{N} 1$ et $\mathrm{N} 2$, le rôle plus ou moins sémantiquement important de N2, même si ce dernier est bien rarement une information nouvelle : il semble difficile de poser l'existence d'un nom et de le qualifier en même temps. Et $\mathrm{N}$ of $\mathrm{N}$ constitue une réorganisation synthétique d'un énoncé où 
N2 est sujet (donc généralement thématique). Ainsi, pour la même structure, deux analyses (au moins) sont possibles suivant les poids respectifs de $\mathrm{N} 1$ et $\mathrm{N} 2$, dont l'une montre une déconnexion partielle forme (tête syntaxique) / sens (noyau sémantique).

En résumé :

1 He's a gem [of a person]: pas de déconnexion, of a person reprenant he et jouant ainsi un rôle secondaire, a gem est la tête syntactico-sémantique au sein de NofN, et constitue l'apport d'information. Of a person permet de construire un type mais ne joue pas un rôle essentiel. He is a gem of a person.

2 Edward the conqueror is [a brilliant gem of] a short story : déconnexion partielle. L'élément sémantique central est d'abord short story puis sa qualification. L'ordre informatif est donc inverse à l'ordre linéaire (l'on a d'abord short story puis a gem, alors que l'ordre linéaire est inverse), et tête syntaxique et noyau sémantique sont en décalage partiel. L'élément sémantique principal est $\mathrm{N} 2$, d'où son impossible omission: Edward the conqueror is a brilliant gem of a short story.

La même structure, qui synthétise un schéma nom qualifié + relative adjectivale qualifiante en en inversant l'ordre, peut donc se décrire de deux façons différentes (au moins) suivant le contexte. Le modèle explicatif peut donc varier. Un élément stable toutefois : of établit à chaque fois un lien d'identification entre les deux $\mathrm{N}$.

Proposer une formalisation qui s'adapte au sens contextuel d'une structure, même si la forme reste la même, correspond à une démarche stylistique.

\section{The Unconsoled. L'éternel retour du discours indirect libre (DIL), or so it seems... Première figure de l'indécidable : récit ou DIL ?}

Dans Le discours indirect libre au risque de la grammaire (2006), j'ai proposé l'hypothèse suivante: dans la mesure où le DIL ne constitue pas une forme syntaxiquement repérable de discours rapporté (DR), contrairement au discours indirect classique (DIC) et au discours direct 
(DD), la reconnaissance du DIL doit être fondée sur sa ressemblance avec un génodiscours reconstruit à travers lui :

Finally, however, the decision came down to pardon and release Hanna, and the governor called me. Could I come now ? Hanna was getting out in a week. (Schlink, The Reader, 1995, 192)

Le directeur de la prison appelle le narrateur pour lui demander de venir chercher Hanna, qui va être libérée. Son énoncé (en gras) est rapporté en DIL: aucune incise ou proposition matrice ne le signale comme tel mais un génodiscours vraisemblable est reconstructible : Could you come now? Hanna is getting out in a week. On constate alors que l'énoncé comporte des marques d'indirection : you $\longrightarrow I$, is $\longrightarrow$ was.

L'on a donc bien affaire à du DIL.

Mais les difficultés commencent lorsque la reconstruction d'un génodiscours est malaisée, voire impossible :

"What's that you've got here, Harry ?" It was Quidditch ${ }^{10}$ through the Ages. Harry showed him. (Rowlings, Harry Potter and the Philosopher's Stone, 2000 [1997], 197)

Le statut du segment en gras pose problème : s'agit-il de la réponse à la question posée en $\mathrm{DD}$ verbalisée par le personnage et rapportée en DIL, le génodiscours correspondant à It is Quidditch through the Ages ? Ou s'agit-il d'un énoncé en récit, imputable au narrateur, le personnage n'ayant fait que montrer le livre mais sans rien dire, comme semble l'indiquer Harry showed him. En d'autres termes, est-il à mettre sur le compte du narrateur (auquel cas on se trouve en récit) ou $\mathrm{du}$ personnage (auquel cas on a affaire à du DIL) ? En raison de la présence de Harry showed him, on penche pour le récit. Mais l'énoncé est indécidable, parce qu'un génodiscours est impossible à poser.

Le récit génère sans cesse de l'indécidable, précisément parce que le DIL n'est pas une forme, et qu'il ne se distingue pas, formellement, du récit. Le contexte parfois ne permet pas de trancher.

On prendra un autre exemple :

10 Un sport du monde des sorciers. 
(point de vue de Peter Walsh) But it was Clarissa one remembered. Not that she was striking; not beautiful at all; there was nothing picturesque about her; she never said anything particularly clever; there she was, however; there she was.

No, no, no! He was not in love with her any more! He only felt, after seeing her that morning [...] unable to get away from the thought of her. (Woolf, Mrs Dalloway, 1947 [1925], 112)

Tout le début jusqu'à there she was relève clairement du récit à point de vue, qui se distingue du DIL en ce qu'on se trouve dans les pensées du personnage, mais aucun génodiscours n'est reconstructible dans ce cas. Mais l'énoncé en gras pose problème : No, no, no! He was not in love with her any more! A un premier niveau on peut croire à du DIL. C'est bien Peter Walsh, toujours amoureux de Clarissa, qui lutte contre cette pensée obsédante. Un fort effet mimétique est produit, venant de l'exclamation, de la répétition de no, évoquant un discours intérieur qui prend la forme d'un discours oralisé. Mais dès que l'on creuse l'analyse, l'interprétation DIL de ce segment est impossible à poser, même si certains indices semblent aller dans ce sens. Si l'on ajoute une incise :

No, no, no! He was not in love with her any more! He thought. He only felt, after seeing her that morning $[. .$.$] unable to get away from the thought$ of her.

L'incise crée un hiatus avec ce qui suit, qui relève clairement du récit à point de vue comme le souligne la présence de $\mathrm{He}$ only felt, qui décrit une perception du personnage par le narrateur. On aurait alors tendance à interpréter le segment comme du récit à point de vue : le narrateur met en mots la pensée du personnage, non nécessairement intérieurement verbalisée, et raconte l'histoire du point de vue du personnage en mimant son expression. C'est ce que Danon-Boileau (1982, 82) appelle 'discours mimétique du narrateur'. Mais une $3^{\text {ème }}$ interprétation est possible : le récit construit un attendu (he's still in love with her), que cet énoncé vient démentir. Dans ce cas, l'énoncé en gras provient du narrateur consonant, qui ne fait plus qu'un avec son personnage. Il s'agit alors d'une intervention du narrateur déguisée.

En d'autres termes, Peter Walsh est incontestablement construit comme repère-point de vue dans cet extrait mais fonctionne-t-il comme locuteur cité, ce qui favoriserait l'interprétation DIL ? Peut-il se dire ceci à ce moment du récit, sachant que les personnages de Mrs Dalloway ne sont 
pas des personnages au sens classique du terme, manquant de profondeur et de contours, car l'on passe sans cesse des perceptions de l'un aux perceptions de l'autre (le génodiscours hypothétique est en gras):

But it was Clarissa one remembered. Not that she was striking; not beautiful at all; there was nothing picturesque about her; she never said anything particularly clever; there she was, however; there she was.

No, no, no! I am not in love with her any more! He only felt, after seeing her that morning [...] unable to get away from the thought of her.

3 interprétations sont donc possibles :

1. récit à point de vue très mimétique (le narrateur s'exprime dans les termes du personnage mais un génodiscours vraisemblable à cet endroit du récit n'est pas reconstructible);

2. intervention du narrateur déguisée,

3. DIL mais l'incise ajoutée fait obstacle.

Cet énoncé est donc indécidable et précisément ce type de littérature moderniste vise à produire des formes indéterminées. Narrateur et "personnage" ne se distinguent plus. Il ne faut donc pas chercher à les distinguer. Et accepter l'irrésolu.

\section{Syntaxe mensongère. Autre figure de l'indécidable : entre DD et DISL (discours indirect semi-libre)}

Le statut énonciatif de nombre d'énoncés rencontrés dans Mrs Dalloway est indécidable, voire trompeur. L'hésitation ne se situe plus entre DIL et récit, comme ci-dessus, mais au sein même des formes de $\mathrm{DR}$, qui deviennent poreuses.

Un exemple: une conversation se déroule entre Rezia Smith, épouse de Septimus Smith, et Sir William, le médecin appelé au chevet de Septimus ; ce dernier est présent une partie de l'échange, ce qui brouille la destination des messages; on ne sait plus par endroits si Sir William s'adresse à Rezia ou à Septimus :

If Mr. Smith would wait, said Sir William, he would speak to Mrs. Smith in the next room. Her husband was very seriously ill, Sir William said. Did he threaten to kill himself?

Oh, he did, she cried. But he did not mean it, she said. Of course not. It was merely a question of rest, said Sir William; of rest, rest, rest; a long rest 
in bed. There was a delightful home down in the country where her husband would be perfectly looked after. Away from her? she asked. Unfortunately, yes; the people we care for most are not good for us when we are ill. But he was not mad, was he? Sir William said he never spoke of "madness"; he called it not having a sense of proportion. But her husband did not like doctors. He would refuse to go there. Shortly and kindly Sir William explained to her the state of the case. He had threatened to kill himself. There was no alternative. It was a question of law. He would lie in bed in a beautiful house in the country. The nurses were admirable. Sir William would visit him once a week. If Mrs. Warren Smith was quite sure she had no more questions to ask-he never hurried his patients-they would return to her husband. She had nothing more to ask-not of Sir William.

[...]

"We have had our little talk," said Sir William.

"He says you are very, very ill," Rezia cried.

"We have been arranging that you should go into a home," said Sir William.

$[\ldots]$

"Impulses came upon him sometimes?" Sir William asked, with his pencil on a pink card.

That was his own affair, said Septimus.

"Nobody lives for himself alone," said Sir William, glancing at the photograph of his wife in Court dress. (144)

Cette conversation est essentiellement rapportée indirectement en discours indirect libre semi-libre (DISL, discours indirect libre suivi d'une incise $)^{11}$, avec quelques fragments de discours direct. Le statut énonciatif de l'énoncé en gras pose problème : à qui est-il adressé ? A Rezia ? Fort probablement, conformément au reste de l'échange. Ce sera notre première hypothèse.

S'agit-il de DD, comme semblent l'indiquer les guillemets?

Si l'on reconstruit un génodiscours, on se rend compte qu'un changement est intervenu :

"Impulses come upon him sometimes?" Sir William asked, with his pencil on a pink card.

En DD, le temps ne serait pas ajusté ; le médecin n'aurait pas de raison d'employer le prétérit dans ce contexte, étant donné qu'il

${ }^{11}$ Le discours indirect semi-libre (DISL) rappelle le DD par sa forme avec incise, frontale, médiane ou finale, mais l'absence de guillemets et les marques d'indirection (repérages temporels et/ou de personnes ajustés) l'en distinguent :

"I'm tired", she said. DD

She was tired, she said. DISL 
s'interroge sur les symptômes présents de Septimus, ce que semble indiquer aussi l'adverbe de fréquence sometimes.

L'emploi du prétérit est la trace d'une narrativisation, d'une indirection dont le narrateur est responsable, ce qui change le statut énonciatif de l'énoncé, malgré la présence des guillemets. Un repérage temporel et / ou un repérage des personnes ajustés sont des traces d'indirection.

En raison de l'ajustement temporel et de la présence de l'incise Sir William asked, l'on a donc affaire à du DISL, déguisé en DD en raison des guillemets. Ce faux DD fait écho au simulacre de dialogue entre Rezia et Sir William, cette dernière n'ayant aucune confiance ni sympathie pour lui en tant que médecin.

Deuxième hypothèse :

Le discours du médecin s'adresse à Septimus, et le génodiscours reconstruit serait alors: "Impulses come upon you sometimes?" Sir William asked.

Deux ajustements ont été effectués : le temps et la personne. On a alors clairement affaire à du DISL déguisé en DD.

Cette hypothèse, moins privilégiée, est cependant en phase avec la réponse, venant de Septimus, et non de Rezia, également donnée en DISL : That was his own affair, said Septimus.

Dans les deux cas, que l'énoncé s'adresse à Rezia avec un ajustement minimal (seulement le temps) ou à Septimus, avec un ajustement complet, on a affaire à de la syntaxe mensongère : un énoncé indirect se présente comme un énoncé direct, en raison des guillemets.

Troisième hypothèse :

On a affaire à du DD, et le prétérit figure dans l'énoncé initial :

"Impulses came upon him sometimes?" Sir William asked, with his pencil on a pink card.

On retombe sur l'énoncé à analyser. Mais cette hypothèse n'est pas privilégiée, sans pouvoir être exclue.

Cette hésitation quant au statut énonciatif de l'énoncé et à son destinataire, due à la présence de formes qui paraissent ajustées, se perçoit également dans les traductions, qui ont fait le choix de la simplification : 
- Avait-il parfois des crises, demanda Sir William, le crayon sur la fiche rose.

C'était son affaire personnelle, dit Septimus. Traduction de Merle et al. 1993, 281.

La traduction fait apparaître un DISL non hybride, sans guillemets. Pas de tiret pour la prise de parole de Septimus, rapportée en DISL.

"Il a quelquefois des accès?", demanda Sir William, le crayon sur la carte rose.

Septimus répondit que cela ne regardait que lui. Traduction de David 2013, 113.

Emploi du présent dans la citation, transformant l'énoncé origine en DD typique, alors que l'énoncé anglais contient un passé (hybridité).

Transformation de la réponse de Septimus initialement en DISL (That was his own affair, said Septimus) en DIC (Septimus répondit que cela ne regardait que lui).

L'utilisation des guillemets donne ainsi l'impression que ce sont les paroles vraies du personnage qui sont rapportées. Mais ceci n'est qu'un leurre car la présence narratoriale est toujours perceptible au niveau du repérage temporel et des personnes. La $3^{\text {ème }}$ hypothèse est très peu probable.

La forme est trompeuse et l'énoncé est ici indécidable. L'on ne peut trancher entre discours de Sir William rapporté en DISL adressé à Rezia (le temps uniquement est ajusté) ou à Septimus (le temps et le repérage des personnes sont ajustés), ou discours de Sir William rapporté en DD (pas d'ajustement dans la citation) adressé à Rezia.

L'analyse stylistique fait une place à l'indécidable, qu'elle ne cherche pas à gommer mais à intégrer. L'indétermination est au cœur du style de Virginia Woolf.

\section{L'irrésolu}

Dans "Le double et l'absent » $(1992,46)$, Green souligne que

Des travaux récents (S. Viderman) nous ont appris que l'interprétation n'est pas seulement la révélation d'un sens caché, mais, d'une certaine 
façon, la création d'un sens absent, une véritable invention d'un sens resté, comme on dit, en souffrance.

Ceci s'applique bien à l'extrait de Mrs Dalloway ci-dessous, qui ne cesse de m'interroger depuis 20 ans :

"But let us sit down for five minutes," said Richard.

It all looked so empty. All the chairs were against the wall. What had they been doing? Oh, it was for the party; no, he had not forgotten, the party. Peter Walsh was back. Oh yes; she had had him. And he was going to get a divorce; and he was in love with some woman out there. And he hadn't changed in the slightest. There she was, mending her dress. . . .

"Thinking of Bourton," she said.

"Hugh was at lunch," said Richard. She had met him too! Well, he was getting absolutely intolerable. Buying Evelyn necklaces; fatter than ever; an intolerable ass.

"And it came over me 'I might have married you'," she said, thinking of Peter sitting there in his little bow-tie; with that knife, opening it, shutting it. "Just as he always was, you know." (Mrs Dalloway, 175)

"Thinking of Bourton, "she said relève syntaxiquement du DD. Il est annoncé comme ayant été verbalisé (she said). Pourtant, il n'a en aucun cas pu l'être dans l'univers de fiction: Bourton est attaché à Peter Walsh, rival amoureux de Richard Dalloway, que Mrs Dalloway a un temps pensé épouser, avant d'accepter la main de Dalloway. Nous sommes au début du $\mathrm{XX}^{\mathrm{e}}$ siècle, dans un milieu où la retenue est de mise. Dire à son mari que l'on pense à un lieu attaché à un rival amoureux relève de l'impensable. L'énoncé se présente comme dit alors qu'il ne peut l'avoir été. Peut-on alors postuler que Mrs Dalloway se parle à elle-même et que l'on rapporte ses pensées ? L'incise She said contrevient à cette hypothèse, car même si said était employé à la place de thought ${ }^{12}$, ce qui est fréquent dans l'écriture de V. Woolf, pourquoi Mrs Dalloway se décrirait-elle en train de penser? Pourquoi se dire, je suis en train de penser à Bourton, lorsqu'on est en train d'y penser ? On y pense, sans le dire... Et le contenu de la pensée ne peut être verbalisé que par le narrateur. Les guillemets sont ici détournés de leur fonction première, qui est de signaler qu'un acte de parole (qui peut être intérieur) a eu lieu et qu'il est rapporté. Cet énoncé

12 Poncharal (2004) souligne que le verbe say est quelque peu désémantisé dans les incises de DIL; il ne rend compte que d'un seul pôle de la communication. L'on peut alors formuler l'hypothèse que say, dans les cas évoqués, a le sens de say to oneself. Cela dit, dans ce cas précis, "Thinking of Bourton", she said to herself ne fonctionne pas non plus : ? "En pensant à Bourton ", se dit-elle. 
relève syntaxiquement $d u$ DD mais au niveau du sens, il ne fait que produire un effet de DD, le discours initial n'étant pas récupérable dans l'univers du récit. Aucun génodiscours n'est reconstructible. Il s'agit de la verbalisation par le narrateur d'une pensée, présenté comme verbalisée alors qu'elle ne peut l'être. L'on a affaire à du récit à point de vue, prenant la forme du DD.

On est dans la pure syntaxe mensongère. Ce passage a d'ailleurs posé problème aux différents traducteurs de l'œuvre. La traduction de Pasquier (1994, 218) par exemple propose : "En pensant à Bourton ", ditelle. L'énoncé conserve en français le même statut d'étrangeté qu'il a en anglais. On le comprend plutôt comme faisant suite à la description du narrateur qui précède (les guillemets et l'incise ont été supprimés, et on a changé la ponctuation afin de le faire apparaître) : There she was, mending her dress, thinking of Bourton.

Les deux énoncés en gras suivants relèvent du même phénomène de syntaxe mensongère. Ils se présentent une fois de plus comme ayant été verbalisés (les guillemets semblent l'attester, ainsi que l'incise she said), et pourtant, dans le contexte, il sont nécessairement intérieurs ou partiellement intérieurs : Mrs Dalloway est en présence de son mari mais elle pense à Peter Walsh, dont elle se dit qu'elle aurait pu l'épouser. C'est à ce dernier que "you " renvoie, et non à son mari, qui est face à elle et auquel elle est déjà mariée ... Elle ne peut donc lui dire "I might have married you ".

En outre, même si cet énoncé est nécessairement intérieur, malgré la présence de she said, il ne peut vraisemblablement avoir été mentalement verbalisé tel quel. Ici encore, le génodiscours ne peut correspondre à "It comes over me "I might have married you" ". Tout au plus peut-elle penser "I might have married you ". Car en effet, lorsque quelque chose vient à l'esprit, il vient à l'esprit, sans que l'on ne se dise "It comes over me".

Si l'on traite ce passage comme s'il avait réellement été dit dans le contexte (ce que semble indiquer la forme), comme si say avait son sens habituel, l'on se méprend sur le sens du passage.

En d'autres termes, comme je l'ai précédemment souligné (De Mattia-Viviès 2010), la forme signifiante < ' $X$ ', Y said > n'a du DD que la forme et une partie du sens qui lui est attaché, le sens 'discours rapporté'. Mais ce sens rapporté peut être détourné si au niveau pragmatique l'énoncé ne correspond pas à un acte verbalisé, intérieurement ou 
extérieurement, que l'univers soit fictif ou non. Le passage a beau relever du DR, si aucun acte ne peut être posé, on interprétera le passage comme du récit du narrateur déguisé en prise de parole du personnage (ici Mrs Dalloway).

Ce détournement des formes de DR crée un sens absent au niveau formel. C'est comme si, de manière détournée, par cette syntaxe mensongère, cet énoncé révélait le fonctionnement du couple Dalloway, et le faisait apparaître pour ce qu'il est : un lieu où l'on croit se parler mais où la parole directe est impossible, où l'essentiel ne peut se dire. La syntaxe mensongère dirait alors la vérité, une vérité que les personnages ne peuvent se dire.

\section{Conclusion. De la rupture dans l'unité du sujet parlant à la rupture dans l'unité du sujet pensant.}

J'ai tenté de donner un aperçu de la méthode employée pour l'analyse de certains faits de langue ou d'extraits de romans, dans l'optique stylistique qui est la mienne et qui se fonde sur une déconnexion possible de la forme et du sens, qui a partie liée avec la notion stylistique d'écart.

Ce parcours stylistique a également débouché sur d'autres investigations correspondant à des intérêts antérieurs, rendues possibles par l'interdisciplinarité, actuellement encouragée par les unités de recherches. La rupture dans l'unité du sujet parlant conduit à analyser la rupture dans l'unité du sujet pensant. Mon intérêt pour la psychiatrie a pris depuis quelques années la forme d'une recherche menée auprès d'enfants qui souffrent de dysphasies (troubles du langage). Grâce à la convention établie entre le LERMA (EA 853), l'unité de recherche à laquelle j'appartiens, et l'hôpital Salvator à Marseille (service du Dr Jean-Luc Di Stefano), et conjointement avec ma collègue Sara Greaves, maître de conférences HDR au département d'études anglophones de l'université d'Aix-Marseille, je tente, à partir de l'observation d'enfants dysphasiques, de comprendre ce qui, au plan psychologique, sous-tend les difficultés d'apprentissage d'une langue (maternelle) en établissant un lien avec les difficultés d'apprentissage d'une langue étrangère. Qu'est-ce qui se joue ou se rejoue lorsqu'on apprend une langue étrangère (en l'occurrence l'anglais) ? Je pose l'hypothèse d'une interférence nécessaire de la langue maternelle dans l'apprentissage d'une langue étrangère afin que ce dernier 
soit réussi, d'un lien à la langue maternelle qui ne doit pas être rompu (ou tenté d'être rompu) à travers cet apprentissage. Cette hypothèse s'est construite à partir des questions suivantes : en quoi la langue maternelle bloque-t-elle ou favorise-t-elle (ou les deux à la fois) l'apprentissage d'une langue étrangère ? La langue étrangère doit-elle être, dans les termes de Foucault, hors matrie, c'est-à-dire le plus possible libérée de la langue maternelle ? Doit-elle et peut-elle être une langue sans reste, débarrassée de l'empreinte maternelle ? Parler l'étranger est-il possible sans devenir étranger à soi-même ? Qu'est-ce que la langue étrangère oblige à quitter ? Une déconnexion de la langue maternelle est-elle possible ? Ces questions, de même que l'hypothèse de travail à laquelle elles ont abouti, sont traitées dans une article à paraître intitulé "Entrer dans la langue ou dans les langues : de la langue maternelle à la langue mat-rangère ".

La stylistique conduirait-elle aux sciences humaines? Dans ce cas, elle constituerait le reste de l'anglistique, dans sa prolifération salutaire, dans son avant-gardisme créateur. La stylistique multiforme, impossible à enfermer dans une définition, lieu où le langage se reflète, se reconnaît et se méconnaît, se fait et se défait, n'est-elle pas le lieu même de l'investigation du linguiste? Toute grammaire ne devrait-elle pas être stylistique, c'est-à-dire fondamentalement en prise avec la langue telle qu'elle est, stable et instable tout à la fois ? Certes, le grammairien stylisticien serait alors mis dans une position tout aussi instable. Mais à cause du langage, par le fait même de l'existence du langage, l'homme, audelà du linguiste, est-il maître en la demeure ? "Who speaks ? Language or the speaker?"13 Ou les deux à la fois, dans une sorte de "compromis freudien "?

L'analyse stylistique offre la liberté suprême de ne pas répondre de manière univoque à ces questions.

13 Lecercle $(1990,5)$. 


\section{CORPUS}

AUSTEN, J. 1813. Pride and Prejudice.

http://gutenberg.net.au/ebooks/m00008.html\#CHAPTER_1, consulté le 2 mars 2018.

FORSTER, Edward Morgan. 1908. A Room with a View.

http://www.literaturepage.com/read/roomwithaview-100.html, consulté le 2 mars 2018.

ORWELL, George. 1989 [1949]. Nineteen Eighty-Four. Londres : Penguin Books.

ROWLINGS, Joanne. 2000 [1997]. Harry Potter and the Philosopher's Stone. Londres : Bloomsbury.

SCHLINK, Bernhard. 2008 [1995]. The Reader. London : Phoenix.

THOMAS, Dylan. 1980 [1955]. "The Followers ». Modern Short Stories in English. Cheltenham : Nelson Thornes.

WOOLF, Virginia. 1947 [1925]. Mrs Dalloway. Londres: The Albatross.

\section{Traductions}

WOOLF, Virginia. 1994 [1925]. Mrs Dalloway. Traduction de MarieClaire Pasquier. Coll. "Folio-Classique ». Paris : Gallimard.

WOOLF, Virginia. 2013 [1925]. Mrs Dalloway. Traduction de Simone David. Paris : Flammarion. 


\section{BIBLIOGRAPHIE ${ }^{14}$}

BAKHTINE, Mikhaïl. 1977 [1926]. Marxisme et philosophie du langage. Paris : Editions de Minuit.

BANFIELD, Ann. 1973. "Narrative Style and the Grammar of Direct and Indirect Speech ». Foundations of Language $10: 1-39$.

CHUQUET, Jean. \& BOUSCAREN, Janine. 1987. Grammaire et textes anglais. Guide pour l'analyse linguistique. Paris : Ophrys.

COATES, Jennifer. 1985 [1983]. The Semantics of the Modal Auxiliaries. Surry Hills, Australia : Croom Helm.

COTTE, Pierre. 1983. "Of et la modification ». SIGMA 7 : 95-113.

COTTE, Pierre. 1996. L'explication grammaticale de textes anglais. Paris : Presses Universitaires de France.

DANON-BOILEAU, Laurent. 1982. Produire le fictif. Paris : Klincksieck.

DE MATTIA, Monique. 2000. Le discours indirect en anglais contemporain. Approche énonciative. Aix-en-Provence : Publications de l'Université de Provence.

DE MATTIA-VIVIES, Monique. 2006. Le discours indirect libre au risque de la grammaire. Entre fixité et mobilité : de la description à l'impossible saisie. Aix-en-Provence : Publications de l'Université de Provence.

DE MATTIA-VIVIES, Monique. 2006. "De la porosité des formes du discours rapporté aux cas de déconnexion forme / sens dans l'univers du récit ». In C. Delesse (dir.), Approche(s) linguistique(s)

${ }_{14}$ Toutes les références citées en bibliographie n'apparaissent pas nécessairement dans le corps de l'article mais elles ont assurément sous-tendu la réflexion. 
et/ou traductologique(s). Université d'Artois: Artois Presses Universités, collection Traductologie, p. 29-52.

DE MATTIA-VIVIES, Monique. 2010. " Du discours rapporté mimétique aux formes intrinsèquement hybrides ". AnglophoniaSigma 28 : 151-180.

DE MATTIA-VIVIES, Monique. 2012. "Syntax in Wonderland. Les déconnexions forme / sens et la syntaxe dite mensongère ". In M. De Mattia-Viviès (dir.), Les déconnexions forme / sens et la syntaxe dite mensongère. E-rea 9.2. URL : http://journals.openedition.org/erea/2651, consulté le 2 mars 2018.

DE MATTIA-VIVIES, Monique. 2018. Leçons de grammaire anglaise. De la recherche à l'enseignement : syntaxe (volume I). (A paraître en septembre 2018, Presses Universitaires de Provence)

DE MATTIA-VIVIES, Monique. 2019. Leçons de grammaire anglaise. De la recherche à l'enseignement : le groupe nominal (volume II). (A paraître en janvier 2019, Presses Universitaires de Provence)

DE MATTIA-VIVIES, Monique. 2019. Leçons de grammaire anglaise. De la recherche à l'enseignement : le groupe prédicatif (volume III). (A paraître en juin 2019, Presses Universitaires de Provence)

DE MATTIA-VIVIES, Monique. 2019. "Entrer dans la langue ou dans les langues : de la langue maternelle à la langue mat-rangère." (Article soumis pour publication).

DELESSE, Catherine (dir.). 2006. Discours rapporté(s). Approches linguistiques et/ou traductologiques. Université d'Artois: Artois Presses Universités, collection Traductologie.

DUCROT, Oswald. 1984. Le dire et le dit. Paris : Editions de Minuit.

FURMANIAK, Grégory. 2015. "A quoi sert la modalisation a posteriori ?". Anglophonia 19, http://anglophonia.revues.org/467. URL : http://journals.openedition.org/anglophonia/467, consulté le 2 mars 2018 . 
GREEN, André. 1992 [1973]. "Le double et l'absent ». La déliaison. Anthropologie, psychanalyse et littérature. Paris: Hachette, coll. "Pluriel ".

HALLIDAY, Michael \& HASAN, Ruqaiya. 2014 [1976]. Cohesion in English. Londres: Routledge.

KIPARSKY, Paul \& Carol. 1971. "Fact». In Steinberg \& Jakobovits (eds), Semantics. Londres: Cambridge University Press, p. 345369 .

LACAN, Jacques. 1981. Séminaire III. Les Psychoses. Paris : Le Seuil.

LARREYA, Paul. 2000. "Connaissance, inférence et modalités épistémiques dans le système verbal de l'anglais ". In Jean Pauchard (dir.), La modalité et les modaux en diachronie et en synchronie (domaine anglais). Reims : Presses Universitaires de Reims, p. 175199.

LARREYA, Paul. 2009. "Towards a Typology of Modality in Language ". In Salkie, Raphael, Pierre Busuttil, and Johan van der Auwera (eds), Modality in English: Theory and Description. Berlin and New York: Mouton de Gruyter, p. 9-30.

LECERCLE, Jean-Jacques. 1990. The Violence of Language. Londres : Routledge.

LECERCLE, Jean-Jacques. 1996 [1990]. La violence du langage. Paris : Presses Universitaires de France.

MC CAWLEY, James. 1973. "External NP vs Annotated Deep Structures ». Linguistic Inquiry 4.2 : 221-240.

PHILIPPE, Gilles. 1997. Le discours en soi. La représentation du discours intérieur dans les romans de Sartre. Paris : Honoré Champion.

PHILIPPE, Gilles. 2005. «La langue, le style, le sens ». In Claire BadiouMonferran, Frédéric Calas, Julien Piat et Christelle Reggiani (dir.), 
Etudes offertes à Anne-Marie Garagnon. Paris: Editions l'Improviste, p. 285-294.

PHILIPPE, Gilles. 2016. French Style. L'accent français de la prose anglaise. Paris : Les impressions nouvelles.

PONCHARAL, Bruno. 2003. "Divergences énonciatives et stylistiques au DIL en anglais et en français ». In Gilles Mathis, Monique De Mattia et Claire Pégon (dir.), Stylistique et énonciation : le cas du discours indirect libre. Numéro spécial du Bulletin de la Société de Stylistique Anglaise. Nanterre : Atelier de Reprographie intégré, p. 249-266.

RIVARA, René. 1979. "La quantification corrélative ». SIGMA 4: 65107.

RIVARA, René. 1990. Le système de la comparaison. Paris : Editions de Minuit.

SORLIN, Sandrine. 2014. La Stylistique anglaise. Théories et pratiques. Rennes : Presses Universitaires de Rennes.

VINCENT-ARNAUD, Nathalie \& SALBAYRE, Sébastien. 2006. L'analyse stylistique : Textes littéraires de langue anglaise. Coll. Amphi 7. Toulouse : Presses Universitaires du Mirail. 